October 2014 Vol.:2, Issue:3

(C) All rights are reserved by Huston et al.

\section{A Death in Infancy: Scene Investigation and Consumer Product Safety Reporting}

Keywords: Forensic Pathology; Infant Death; Asphyxia; Scene Investigation; Consumer Product Safety

\begin{abstract}
The authors present a case of asphyxia of an infant who was placed in an infant swing for the night. The parent's original story of the circumstances of the death does not correspond with the physical findings at the time of autopsy. This case reviews the guidelines and importance of infant death scene investigation and procedures to report misuse or malfunction of a consumer product.
\end{abstract}

\section{Journal of Forensic Investigation}

\author{
Butch Huston*, Michael McGee, Kelly Mills, Victor \\ Froloff \\ Ramsey County Medical Examiner's Office, St. Paul, Minnesota, USA \\ Address for Correspondence \\ Dr. Butch Huston MD, Ramsey County Medical Examiner's Office, St. \\ Paul, Minnesota, USA, Tel: 651-266-1700; Fax: 651-266-1720; E-mail: \\ butch.huston@co.ramsey.mn.us \\ Copyright: (c) 2014 Huston B, et al. This is an open access article \\ distributed under the Creative Commons Attribution License, which \\ permits unrestricted use, distribution, and reproduction in any medium, \\ permits unrestricted use, distribution, and
provided the original work is properly cited. \\ Submission: 19 September, 2014 \\ Accepted: 07 October, 2014 \\ Published: 09 October, 2014 \\ Reviewed \& Approved by: Dr. Long Jin, Assistant Professor of \\ Clinical Pathology at Louisiana State University Health Science Center, \\ USA
}

\section{Case Presentation}

An eight month old infant was found by her mother at 5:30 in the morning after placing the infant to bed on a blanket on the living room floor of the family's apartment the night before. The infant was unresponsive but transported to the local hospital and pronounced. The scene investigation revealed a mattress on the floor which was reportedly used by the parents. In the corner of the room was a crib filled with diapers, stuffed animals and extra blankets. A thick blanket was noted on the floor next to the mattress which the mother indicated was used by the decedent to sleep. The father, interviewed separately, stated that the infant was sleeping on the edge of the mattress next to the mother. Formula was noted in the refrigerator and an older infant type swing was noted in another room.

An autopsy revealed several external marks on the anterior neck and on the posterior upper arms (Figures 1,2 and 3).

The internal examination did not reveal any natural disease processes or significant injury. A full body x-ray did not reveal any evidence of acute or remote bony injury. Postmortem toxicology revealed a negative ethanol and drug screen. Vitreous electrolytes were unremarkable for postmortem values. Microbiologic cultures of the lungs were positive of oropharyngeal flora. Cultures of the spleen and nasopharyngeal viral cultures were negative. Microscopic examination of the organ systems did not reveal any significant pathologic abnormalities.

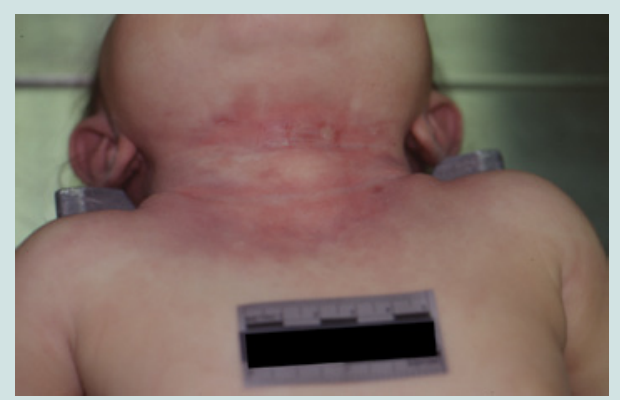

Figure 1: View of the anterior neck revealing abrasions and hyperemic marks.

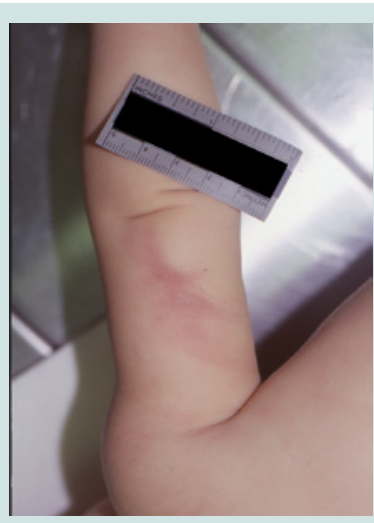

Figure 2: View of the posterior aspect of the right upper arm showing a hyperemic mark

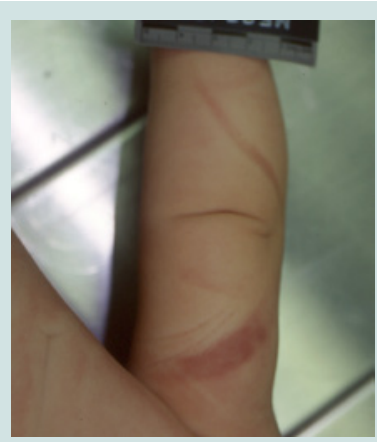

Figure 3: View of the posterior aspect of the left arm showing a hyperemic mark.

\section{Discussion}

The parent's original statements conflicted regarding where the infant was sleeping and found unresponsive. The autopsy revealed several marks on the infant's arms and neck (Figures 1-3) which would indicate that the infant was not sleeping on the blanket, and probably not sleeping on the mattress next to the mother. When re- 
questioned, the parents admitted that they regularly placed the infant in a swing to sleep at night. When the mother woke, she found the infant caught in the waist strap. The infant swing was given to the parents by the maternal grandparents who bought it in a garage sale. Close inspection of the swing revealed that the crotch strap was absent (Figures 4,5). This allowed the infant to partially slip under the waist strap, which resulted in a hanging position by the neck and upper arms (Figure 6). The waist strap corresponded to the marks on the posterior upper arms and the buckle corresponded to the abrasion on the anterior neck (Figure 7).

There is currently no uniform system for the investigation of infant and child deaths in the United States. However, there have been several recommendations have been published [1-4]. Proper infant death investigation consists of a scene investigation, review of the infant's medical history and circumstances leading up to the death and a complete postmortem with toxicology and histological examination. Medical records of the infant's birth, clinic visits and hospitalizations should be obtained and reviewed. A history of past illnesses may provide clues as to the infant's overall health status, possible immune status and the cause of death. Well child visits are important in evaluating the infant's motor, verbal and physical development. The autopsy height, weight and head circumference should be compared to the infant's clinical measurements to determine

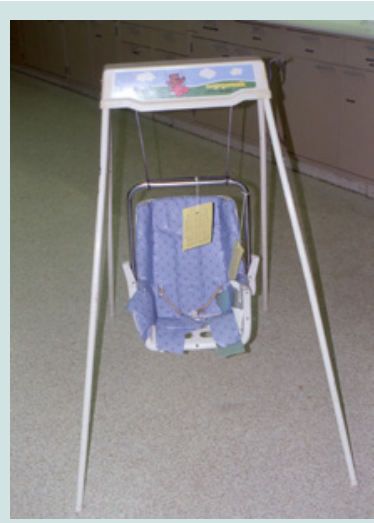

Figure 4: Photograph of the infant swing that the infant was regularly placed in to sleep at night.

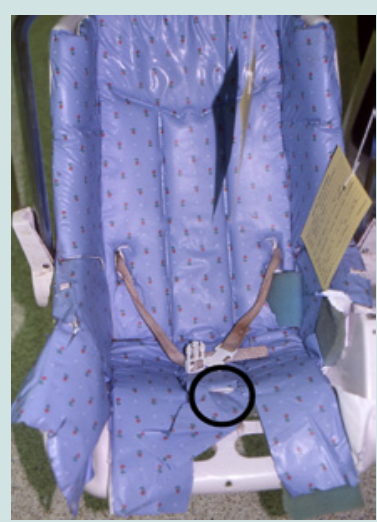

Figure 5: Closer view of the infant swing. The black circle indicates where the crotch strap should be located to form the three point restraint.

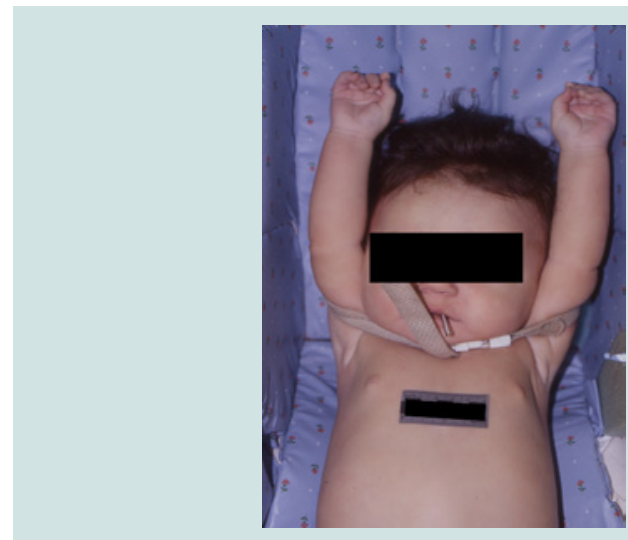

Figure 6: The infant is placed in the swing showing the suspended position of the infant in the swing when it was found.

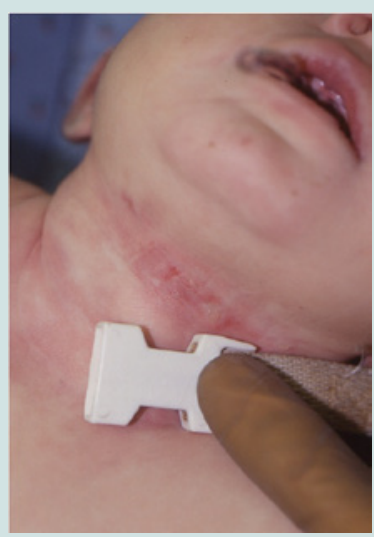

Figure 7: Closer view of the anterior neck showing the infant's anterior neck abrasions and the corresponding buckle/snap of the waist strap.

if the child is on a consistent growth curve. The single measurements obtained at the time of the autopsy cannot be fully interpreted without previous measurements to compare. The birth metabolic screen results should be obtained and reviewed to determine the possible presence or absence of metabolic disorders. If, for whatever reason, a birth screen was not performed, a sample of postmortem blood may be obtained and sent for metabolic screening. Histological analysis of tissues may also assist in ruling out the major types of metabolic diseases. Genetic testing for inherited cardiac anomalies in cases of sudden unexpected infant deaths can be performed but the high cost for these tests often presents a financial difficulty for most Medical Examiner's Offices $[5,6]$.

The circumstances leading the infant's death are important and can be compared with findings obtained from the scene investigation and autopsy. Confirmation of when the infant was last fed may be determined by inspecting the stomach contents and identifying a partial bottle of formula on the nightstand. Obtaining information from multiple individuals at the scene of death may result in corroboration or conflicting stories which may need to be resolved. The caretaker should be asked questions regarding feeding and urine/ stool output. Infectious disease processes in infants may result in subtle clinical symptoms such as decreased appetite or change in feeding, a change in frequency or consistency of stool and urine. 
The importance of changes in breathing may not be recognized by inexperienced parents. Direct questions for the presence of costal retractions, nasal flaring or grunting during respiration should be asked as that information may not be volunteered. Parents and caretakers should be asked about risk factors for SIDs, which include tobacco use in the residence, pets, recent illnesses and sleeping position/arrangement.

The scene should be investigated and documented, either by the medical examiner/coroner, law enforcement or a trained death scene investigator depending on the type of death investigation system in all cases of infant death. There are multiple resources available to guide an investigator during the process. One such resource is the Centers for Disease Control and Prevention (CDC) http://www.cdc.gov/sids/SceneInvestigation.htm\#forms. The CDC website contains a downloadable investigation reporting form which includes witness questions/information, infant medical history, infant dietary, maternal pregnancy history, diagrams and a summary for pathologists. The form, devised in 1999 was revised in 2006 to include new questions regarding SIDs risk factors and is organized to allow easy, consistent data collection and documentation. The form can be modified to fit the needs of individual jurisdictions. Additional in depth investigative forms are also available to download and include an EMS interview, hospital interview immunization record, exposure history, collections log, law enforcement interview and other responder/parental information. Many states and counties have Child Mortality Committees that review infant and child deaths. This form or one similar, makes the collection and compilation of data more consistent over various jurisdictions. The CDC also provides opportunities for training http://www.cdc.gov/sids/TrainingMaterial. $\mathrm{htm}$. The CDC reporting form is very useful regarding deaths involving sleeping conditions but additional questions are necessary if the death involves a circumstance other than sleeping conditions. The scene investigation should be performed as soon as possible, prior to alteration. If the body is transported to the hospital, the scene where the body was initially discovered and transported from should be inspected and documented. Sometimes it is useful to have law enforcement respond to hold the scene or obtain initial photographs.

A part of the scene investigation can be the use of a doll to simulate the position of the infant was last placed in to sleep and the position the infant was found in [4]. Not only is the position of the doll important, but also the position of other nearby items such as blankets, toys and other persons co-sleeping with the infant. The person or provider who placed the infant to sleep should be asked to perform a reenactment as to how the infant was placed. The person or provider who found the infant unresponsive should be the person to perform the reenactment as to the position of the infant when it was found. Some circumstances of death may not require the use of doll reenactment, or may be difficult to recreate. These may include deaths resulting from drowning or falls from heights. There may be times when parents or caregivers are too distraught to provide an accurate recreation of the infant's position. In some instances, untruthful or misleading information from the caregiver, as in this case, may result in an initial inaccurate reenactment, which may only be corrected with additional information from the scene and postmortem examination. Interpretation must be guarded when a caretaker's history of events appears inconsistent, when details change during an interview or when the history given does not appear consistent with other findings.

A complete postmortem examination including full body radiographs should be performed and can include toxicology for alcohol, drugs, and vitreous electrolytes. Microscopic samples should be obtained of the major organ systems and may including the neck organs and multiple samples of cerebral tissue [7,8]. Microbiology cultures can include tissue cultures of the lungs, spleen, blood, cerebral spinal fluid and brain depending on the clinical circumstances and postmortem findings. Nasopharyngeal or direct lung viral cultures may be obtained, especially during portions of the year when viral illnesses are more common. Culture results should be correlated with the clinical circumstances and histological examination. Additional special dissections can be performed including spinal cord, middle ears and eyes depending on the clinical circumstances or history. Areas of injury may be sampled for histological dating purposes if necessary.

If a product is associated with an infant or child's death, that death should be reported to the Consumer Product Safety Commission (CPSC) http://www.cpsc.gov. The CPSC collects information regarding injuries or deaths resulting from consumer products. The CPSC was formed to protect consumers from products that pose a fire, electrical, chemical, or mechanical hazard or can injure individuals including children. Types of products of interest to the Commission include toys, cribs, power tools, cigarette lighters, and household chemicals. The Commission's website includes an online reporting form http://www.saferproducts.gov. The website contains product alerts and can also be used to follow recalled products. This site can also be used to search reported deaths and injuries by product code from the Commission's database. However, the database contains very limited retrievable information online.

\section{References}

1. Kairys SW, Alexander RC, Block RW, Everett VD, Hymel KP,et al. (1999) American Academy of Pediatrics. Committee on Child Abuse and Neglect and Committee on Community Health Services. Investigation and Review of Unexpected Infant and Child Deaths. Pediatrics 104:1158-1160.

2. Centers for Disease Control (1996) Guidelines for Death Scene Investigation of Sudden, Unexplained Infant Deaths: Recommendations of the Interagency Panel on Sudden Infant Death Syndrome. MMWR 45: RR-10.

3. Kaplan SR, Granik LA, ed. (1991) Child Fatality Investigative Procedures Manual. Chicago, IL: American Bar Association.

4. O'Neal BJ (2007) Investigating Infant Deaths. Boca Raton, FL: CRC Press, Taylor \& Francis Group

5. Van Norstrand DW, Ackerman MJ (2010) Genomic Risk Factors in Sudden Infant Death Syndrome. Genome Med 2: 86.

6. Boczek NJ, Tester DJ, Ackerman MJ (2012) The Molecular Autopsy: An Indispensable Step Following Sudden Cardic Death in the Young? Herzschrittmacherther Elektrophysiol 23: 167-173.

7. Bvard RW, Carmichael E, Beal S (1994) How Useful is Postmortem Examination in Sudden Infant Death Syndrome? Pediatr Pathol 14: 817-822.

8. Valdes-Dapena, Marie (1993) Histopathology Atlas for the Sudden Infant death Syndrome. Armed Forces Institute of Pathology. Washington, DC. 